\title{
The Cause Analysis on the Highway Bridge Expansion Joints and the Maintenance of Construction Management
}

\author{
Huadong Li \\ Changchun Architecture and Civil Engineering College Jilin Changchun China 130607 \\ 103285329@qq.com
}

Keywords: Highway Bridges; Expansion joints; Reasons; Maintenance and construction

\begin{abstract}
The bridge expansion joints is to adapt to the structure deformation of the bridge, the across device between the bridge structure each couplet of the beam end and the beam end and the abutment back set free deformation. Its role is to ensure that the bridge span structure under temperature change, concrete shrinkage and creep, load according to its static scheme under the influence of factors such as free expansion and deformation, to make the car comfortable, smooth, and waterproof, prevent mud debris into the seam. This paper mainly discusses for the types and their advantages and disadvantages of highway bridge expansion joints as well as expansion joints, and discusses the preventing and curing of diseases.
\end{abstract}

\section{Introduction}

In recent years with the rapid development of economy, all kinds of infrastructure construction in China ushered in the high speed development stage; Highway bridge project in particular, at the same time, people is becoming more and more attention to highway Bridges safety and life; The main factors are affecting the safety of the highway bridge construction in the construction are produced by the concrete in its shrinkage crack. Under the existing technology, the function of bridge expansion joints can better solve the problem, and has advantages of simple construction, low cost. The bridge expansion joints is located at the upper end, bridge deck broken seam structure activities, mainly by the force bearing system and the displacement of the control system. Bridge expansion joints is the main function: 1) The transfer vehicle through vertical and horizontal load bearing structure to the bridge beam body, 2) It is to adapt to the change of longitudinal and lateral displacement of the bridge and the Angle changes of beam end warp, to ensure the bridge superstructure in the temperature variation, concrete shrinkage and creep. 3) It is to prevent vibration. 4) It is waterproof and drainage. This paper is combined with many years' working experience of the author, a detailed analysis of quality control in the bridge expansion joints construction.

\section{The Importance of Highway Bridge Expansion Joints}

Function lies in the regulation of highway bridge expansion joints, by vehicle load environment characteristics and the physical properties of bridge construction material caused by the displacement between the upper structure and the connection between the upper structures. Bridge expansion joints device is part of the bridge structure, if the improper design and installation of the inferior quality, lack of scientific and timely maintenance, will cause jump in bridge expansion joints. Jumping at bridge expansion joints is the present domestic highway is one of the more common diseases, with the development of highway in our country the problem more and more outstanding performance. Seams bridge expansion joints damage, subsidence, damage of road surface, the different high and low fault (steps), these steps, light the vehicle through a beating and impact, thus to cause additional damage to Bridges and roads load, and make the passenger discomfort, serious and even cause traffic accidents, which affect the normal operation of the highway. Vehicle bridge expansion joints not only in the highway in our country, and outside is also unresolved problems, in order to eliminate the steps, to prevent vehicle, keep good road conditions, the relevant departments and the construction unit adopted many effective measures and methods, 
moving not escape largely reduces the vehicle bridge expansion joints. Bridge expansion joints of the installation construction of highway Bridges is a very important construction projects, installation quality directly affects the driving stability and comfort, service quality and the service life of the bridge. Expansion joints of reasonable selection and installation quality, to ensure the highway transportation is very important for the safe, rapid, comfortable, and directly affect the service life of the bridge and highway in the economy.

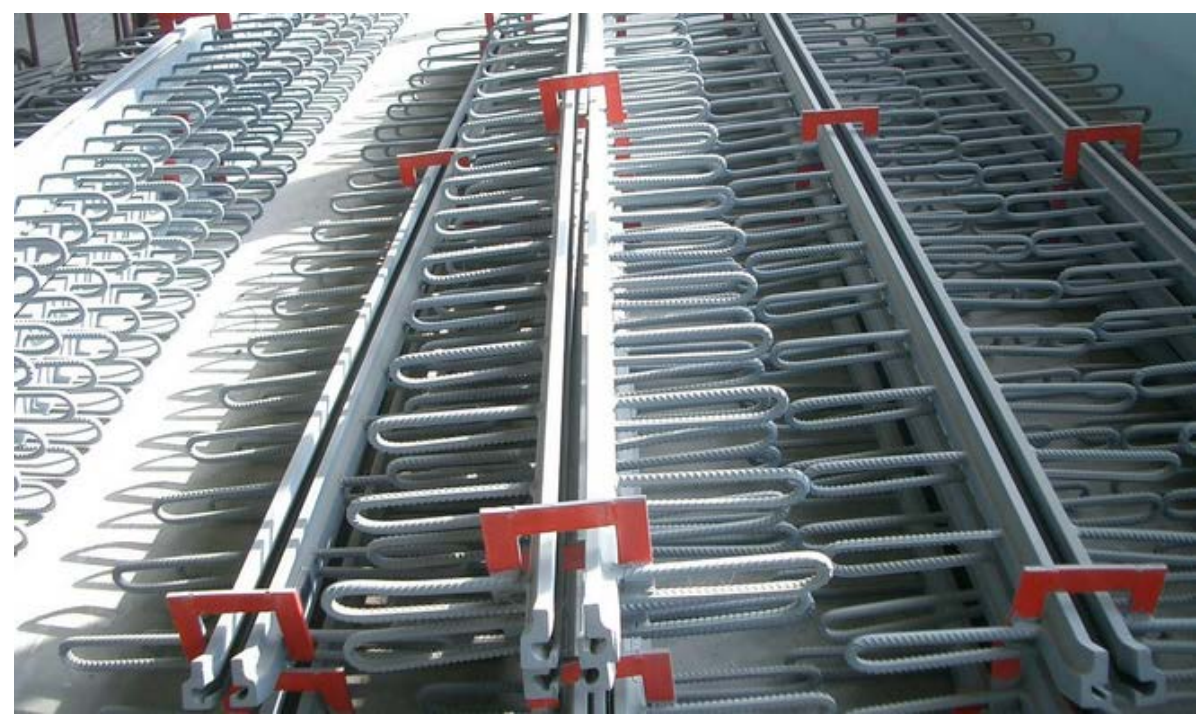

Figure1 the Highway Bridge Expansion Joints Device

\section{The Function and Type of Bridge Expansion Joints.}

The role of bridge expansion joints. The bridge scalability for highway Bridges important influence overall performance and service life. With the increase of traffic, highway Bridges quality enhances unceasingly, the bridge expansion joints function of highway Bridges is more and more prominent. Specific performance in: 1) Reduce the number of traffic accidents. , under the influence of changes in the weather, and the load of highway Bridges in the bridge span structure easily happened displacement of bridge expansion joints can effectively to ensure the stability and comfort of vehicle operation, and effectively reduce the traffic safety accidents. 2) The outside impact cushioning effect, thus protecting highway. Highway Bridges in use process, vehicles will produce strong repeated impact, bridge expansion joints can be directly bear the load of the wheel, a direct hit from a cushion against the impact of highway Bridges. 3) To ensure the safety of the highway Bridges, and ensure the stability of beam body. Reasonable position to set expansion joints, it is in order to make the bridge can adapt to the length of the beam scale changes, so that the beam body is in steady state, ensuring traffic safety and stability.

The type of bridge expansion joints. Currently, the main types of bridge expansion joints in highway Bridges with steel plate type expansion joint, filling type expansion joint, TST crushed stone elastic expansion joint, plate rubber expansion joint, etc.

\section{The Telescopic Device Requirements and the Factors Influencing the Scale}

The overall demand analysis. In highway bridge expansion joints construction control, overall management control system to fully form. For road and bridge expansion device has been installed, to its damage situation in-depth investigation and record, and make comprehensive analysis. The overall requirements of the device for the expansion joint is: to fully to meet the needs of the temperature change of the highway Bridges, able to adapt to bridge caused by the deflection change deflection phenomenon, form the relatively good structure, with high durability and rigidity, and can be fully for drainage and waterproof, does not bring the trouble to the whole construction and maintenance is also relatively simple. 
The influencing factors of scale analysis. In highway bridge construction, there are two main factors influencing the scale of, one is the change in temperature, the second is the shrinkage of concrete. Among them, the temperature change is an important factor, in highway Bridges structure change factors, mostly due to the effects of temperature change, therefore, for the brick, steel bar in concrete, etc, and to form a good proportion, according to local actual temperature to determine the pattern of concrete structure. In the shrinkage of concrete, to involve the water-cement ratio, aggregate and structure geometry size must have comprehensive scientific, fine data, to form the overall expansion amount control better.

\section{The Maintenance Construction Management Measures of the Bridge Expansion Joints}

In view of the above introduction of expansion joints are easy to appear problem, need to strengthen in the process of expansion joint construction for control of construction process, to ensure the sealing, smooth, and has very good strength of concrete, for the use of the expansion joint of rubber material selection need through national certification of products to ensure its quality. To design the structure of expansion joint at the same time need according to the temperature of different structure forms and combined with local conditions rational scale and structure design technology, for the work of bridge inspections should be strengthened at the same time, the timely processing loose screws, repair the damaged road surface, thus ensuring the normal operation of the highway bridge.

The control the construction quality. Due to the large temperature difference, vehicle braking, bridge deck longitudinal slope, factors such as concrete expansion, expansion joints will produce many diseases, including joints of concrete falls off and early destruction, and so on and so forth. In case those facts, not only can make very bumpy, in the process of vehicle engineering overall level to drop, and can also affect the pedestrian safety. Expansion joints appear after damage will produce water seepage phenomenon, this not only makes the beam body erosion, also will rust bearing, shrink beam body can not be normal, lead to the girder design stress under stress is much smaller than the structure of the whole structure of the project. In order to avoid this kind of situation should be to control construction quality, to ensure the overall quality of engineering and compactness.

1) Before the construction. Requirements after the completion of the department of engineering construction in bridge deck paving may; Must choose high quality cement and aggregate, and pass by index test; Must check the quality of expansion joint, expansion joints shall not be used for bending twist; Installing a locator, must be met when the highest temperature expansion joint work with a certain width, the lowest temperature cannot exceed driving requirements when the largest gap. 2) The construction process control. In bridge construction, pay attention to the embedded and reserved, related to the expansion joint installed with accurate installation, welding strong requirements; When installation choice under the environment of low temperature deviation; Installation time to clean up the bridge end gap within the sundry, especially the rabbet parts to wash to clean; After concrete pouring should be continuous, casting to override sprinkler maintenance after reaching a certain intensity recalculate traffic; Fast setting, high strength concrete, should be adopted to offset the anchoring strength of concrete form of shrinkage crack and improve the compactness of concrete permeability in anti vibration. 3) The control of concrete construction. Concrete should not only meet the design strength and good workability, Vibrating wants even when fully and avoid leakage of vibration; Take protective measures to prevent pollution of concrete pavement; we choose sprinkler and keeping in good health with the plastic mulch, during the curing period make-up water every day to keep enough moisture, prevent concrete surface cracks.

To give priority to the new expansion joint. The construction of bridge expansion joint is very important in the process of the whole bridge construction, the quality of expansion joint construction quality will directly affect the quality of the overall structure of the highway bridge construction, expansion joints, so to speak, to a certain extent is directly affecting the stability of bridge structure; So the construction department to elaborate design of bridge expansion joint construction, do a good job in construction management, make the expansion joints, effectively 
guarantee the construction quality, and increase with the increase of vehicle load and traffic volume, the highway bridge construction process should make priority for the new type of expansion joints, which not only can make the time limit for a project can shorten the effective control of engineering quality. Each of the highway bridge construction way is different, so when choosing expansion joint, should according to the actual situation of project selection, give priority to the new, improved expansion joints.

The expansion joint device reasonable choice. Impact on the overall structure of the Bridges, roads and other projects the larger is the quality problem of the expansion joint device, if not reasonable to choose of expansion joints, will directly influence on its use effect, so in the expansion joint device when the choice, should be based on the actual situation to choose the appropriate materials. Selecting the expansion joint, the first factor is the quality and stiffness should be considered, in addition the following aspects should also be considered if durability is whether the machine can effectively resist collision and wear to prevent waste and rain infiltration is able to guarantee smooth vehicles whether exercise can ensure the displacement between the beam and plate, etc.

To strengthen construction process control. The expansion joint is an indispensable part of highway bridge structures. In the process of construction, the stand or fall of expansion joints would have a huge impact on the whole project, in order to ensure that don't appear any problems in the process of engineering use, must strengthen the control of construction engineering, such as expansion joints installation quality control, etc. Strengthen highway bridge expansion joints construction quality control, should be reserved, buries the attention of expansion joints, installation of expansion joint, should pay attention to the welding sequence, and should choose when temperature is low. And before installation, should be to wash and clean up of notches, must clean up the dirt from the gap.

\section{Conclusion}

In the highway bridge construction, the expansion joints setting is beneficial to improving the bearing capacity of the whole bridge project, but if the effect of various factors result in the damage, that will lead to the vehicle in the process of driving the car jumping, hardly conducive to driving safety and comfort, so we must attach importance to the expansion joint setting, through the reasonable design, the careful construction, the strict control to ensure the construction quality, so as to improve the construction quality of bridge engineering and social and economic benefits.

\section{References}

[1] Yanjun Xie, Liantong Mo, Donglin Su, M.F. Woldekidan, Shaopeng Wu. Investigation into fundamental properties of bituminous plug expansion joint filling mixtures containing rubber granules. Construction and Building Materials. 2013(02).

[2] B. Zuada Coelho, A.H.J.M. Vervuurt, W.H.A. Peelen, J.S. Leendertz. Dynamics of modular expansion joints: The Martinus Nijhoff Bridge. Engineering Structures. 2013(11).

[3] Liantong Mo, Donglin Shu, Xun Li, M. Huurman, Shaopeng Wu. Experimental investigation of bituminous plug expansion joint materials containing high content of crumb rubber powder and granules. Materials and Design. 2012(08).

[4] Ramesh B. Malla, Brian J. Swanson, Montgomery T. Shaw. Laboratory evaluation of a silicone foam sealant bonded to various header materials used in bridge expansion joints. Construction and Building Materials. 2011(11). 